\title{
Genetically Determined Serum 25-Hydroxyvitamin D Is Associated with Total, Trunk, and Arm Fat-Free Mass: A Mendelian Randomization Study
}

\author{
R. Kirwan ${ }^{1}$, M. Isanejad ${ }^{2}$,I.G. Davies ${ }^{3}$, M. Mazidi ${ }^{4}$

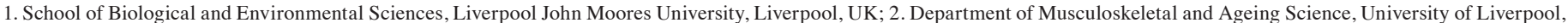

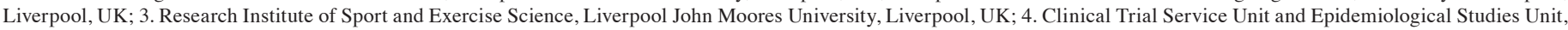 \\ Nuffield Department of Population Health, University of Oxford, Oxford, UK

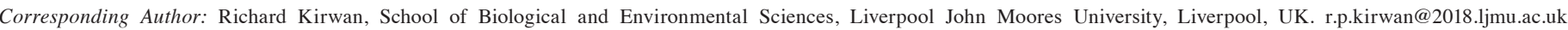 \\ https://orcid.org/0000-0003-4645-0077
}

\begin{abstract}
PURPOSE: Low serum vitamin D status has been associated with reduced muscle mass in observational studies although the relationship is controversial and a causal association cannot be determined from such observations. Two-sample Mendelian randomization (MR) was applied to assess the association between serum vitamin D $(25(\mathrm{OH}) \mathrm{D})$ and total, trunk, arm and leg fat-free mass (FFM).

METHODS: MR was implemented using summary-level data from the largest genome-wide association studies (GWAS) on vitamin $\mathrm{D}(\mathrm{n}=73,699)$ and total, trunk, arm and leg FFM. Inverse variance weighted method (IVW) was used to estimate the causal estimates. Weighted median (WM)-based method, and MR-Egger, leave-one-out were applied as sensitivity analysis.

RESULTS: Genetically higher serum 25(OH)D levels had a positive effect on total $($ IVW $=$ Beta: $0.042, \mathrm{p}=0.038)$, trunk $(\mathrm{IVW}=$ Beta: $0.045, \mathrm{p}=0.023$ ) and arm (right arm IVW = Beta: $0.044, \mathrm{p}=0.002$ left arm IVW = Beta: $0.05, \mathrm{p}=0.005)$ FFM. However, the association with leg FFM was not significant (right leg IVW = Beta: 0.03, p $=0.238$; left leg IVW = Beta: $0.039, \mathrm{p}=0.100)$. The likelihood of heterogeneity and pleiotropy was determined to be low (statistically non-significant), and the observed associations were not driven by single SNPs. Furthermore, MR pleiotropy residual sum and outlier test did not highlight any outliers.

CONCLUSIONS: Our results illustrate the potentially causal, positive effect of serum $25(\mathrm{OH}) \mathrm{D}$ concentration on total, trunk and upper body appendicular fat-free mass.
\end{abstract}

Key words: Muscle mass, Mendelian randomization, 25-Hydroxyvitamin $D, 25(\mathrm{OH}) D$, lean mass, fat-free mass.

\section{Introduction}

$\mathrm{V}$ itamin D is an essential nutrient for human health with roles in multiple biological pathways and low vitamin D status is associated with multiple chronic diseases (1) as well as being associated with musculoskeletal health $(2,3)$ highlighting this nutrient's significance in the global burden of disease. However, up to $40 \%$ of the European population may suffer from vitamin D insufficiency (serum 25 -hydroxy vitamin $\mathrm{D}[25(\mathrm{OH}) \mathrm{D}]$ concentration $<50 \mathrm{nmol} / \mathrm{L}$ ) (4) and vitamin D deficiency $(25(\mathrm{OH}) \mathrm{D}$ concentration $<30$ $\mathrm{nmol} / \mathrm{L}$ )is widespread enough to be considered a global health issue (4-6).
Loss of muscle mass directly affects muscle strength and physical function and as such, sarcopenia, the progressive loss of muscle mass and strength in aging, and frailty (7, 8). Furthermore, muscle mass loss has been associated with a multitude of chronic conditions including cardiovascular disease (CVD) (9), type 2 diabetes mellitus (T2DM) (10), increased risk of falls and fractures (11), cognitive decline and depression $(12,13)$, and all-cause mortality (14). Older adults may spend more time indoors due to poor mobility/reduced muscle function which can further lead to an elevated risk of vitamin $\mathrm{D}$ inadequacy $(15,16)$, leading to a vicious cycle of vitamin D deficiency and loss of muscle mass.

Epidemiological studies suggest an association between low vitamin D status and reduced muscle mass $(3,17,18)$ although some studies have found no such association (19, 20). However, such studies are limited as observational data cannot determine whether an association is causal. Mendelian randomization (MR) analysis uses functional polymorphisms (single nucleotide polymorphisms (SNPs)) associated with specific changes in exposures (in this case, serum $25(\mathrm{OH})$ D) as genetic instruments to determine whether the risk factor is a cause of the disease (21). A major advantage of MR analysis is that they are considerably less prone to confounding, residual bias, and reverse causation than conventional riskfactor epidemiology (22). MR analysis may also circumvent the financial, logistical and ethical limitations of randomised controlled trials (RCTs) and additionally, the data from such studies can inform the design of pilot RCTs and clinical trials by providing information for the potential magnitude of effect of nutrients on a given outcome in specific populations (23).

In the present study, we used MR analysis to determine whether a potential causal relationship exists between serum $25(\mathrm{OH}) \mathrm{D}$ concentration and total, trunk, arm and leg fat-free mass (FFM).

\section{Methods}

\section{Study design}

A two-sample MR study design was used. In a 2-sample MR, the ssummary statistics are provided from various studies for the association of the genetic instruments with the exposure and 


\begin{tabular}{|c|c|c|c|c|c|c|c|}
\hline SNP & Nearest gene & GX & GX SE & EA & OA & EAF & p-value \\
\hline rs3755967 & GC & -0.089 & 0.0023 & $\mathrm{~T}$ & $\mathrm{C}$ & 0.28 & 4.74E-343 \\
\hline rs 12785878 & NADSYN1/DHCR7 & 0.036 & 0.0022 & $\mathrm{~T}$ & $\mathrm{G}$ & 0.75 & $3.80 \mathrm{E}-62$ \\
\hline rs 10745742 & AMDHD1 & 0.019 & 0.002 & $\mathrm{~T}$ & $\mathrm{C}$ & 0.4 & $2.10 \mathrm{E}-20$ \\
\hline rs 17216707 & CYP24A1 & 0.026 & 0.0027 & $\mathrm{~T}$ & $\mathrm{C}$ & 0.79 & $8.14 \mathrm{E}-23$ \\
\hline
\end{tabular}

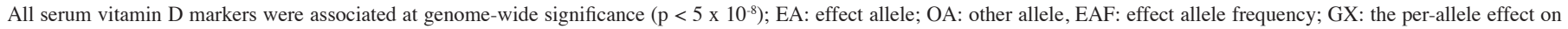
standard deviation units of the telomere length; GX SE: standard error of GX.

outcome. In our study, we obtained the summary statistics from the largest genome wide association studies (GWAS) on serum 25(OH)D (exposure (24)) and FFM (outcome). We applied methods to estimate the unbiased effect of serum $25(\mathrm{OH}) \mathrm{D}$ on FFM (total, trunk, arms and legs,).

\section{Genetic predictors of exposures}

We used six SNPs identified to be associated with circulating $25(\mathrm{OH}) \mathrm{D}$ concentration by the SUNLIGHT meta-GWAS, which are samples of European ancestry (79,366 discovery samples and 42,757 replication samples) (Table 1). GWAS were performed within each cohort according to a uniform analysis plan. Additive genetic models using linear regression on natural-log-transformed $25(\mathrm{OH}) \mathrm{D}$ were fitted and a fixedeffects inverse variance weighted (IVW) meta-analysis across the contributing cohorts was performed (24).

\section{Association of genetic instruments with outcome}

SNPs associated with bioelectrical-impedance-measured fat mass and total, trunk, arm and leg FFM were obtained from analyses by Neale Lab (http://www.nealelab.is). We retrieved the association of the six genetic instruments with SNPs associated with bioelectrical impedance measured FFM using data obtained from UK Biobank. Detailed descriptions of the methods used to measure body composition is available on the UK Biobank website (25). Briefly, whole body as well as site-specific (trunk, leg, arm) fat-free mass/fat mass were evaluated with bioelectrical-impedance analysis (Tanita BC418MA body composition analyser). Body composition of a subset of participants was also assessed using dual-energy X-ray absorptiometry (DXA) which showed high correlation with bio-impedance values (fat-free mass: $r=0.96)(25)$. The UK Biobank is a population-based cohort of approximately 500,000 individuals; $54 \%$ are female, the average age is 57 (range 37-73), while 94\% report as being White British. Further details on the rationale, design and methodology for UK Biobank can be found elsewhere (26).

\section{Mendelian Randomisation analysis}

We combined the effect of six instruments using inverse variance weighted (IVW) method as implemented in Two
Sample MR package of the statistical software, $\mathrm{R}$ ( $\mathrm{R}$ Core Team, Vienna, Austria. https://www.R-project.org/). We assessed the heterogeneity using Q value for IVW. To address the potential effect of pleiotropic variants on the final effect estimate, we conducted sensitivity analysis including weighted median (WM) and MR-Egger. Sensitivity analysis was conducted using the leave-one-out method. The weighted median (WM) estimate, as the weighted median of the SNPspecific estimates, provides correct estimates as long as SNPs accounting for $\geq 50 \%$ of the weight are valid instruments. WM MR allows some variants to be invalid instruments provided at least half are valid instruments. It uses inverse variance weights and bootstrapping to estimate confidence intervals (CIs) (27). MR-Egger has an ability to make estimates by assumption of all SNPs are invalid instruments as long as the assumption of instrument strength independent of direct effect (InSIDE) is satisfied (27). MR-Egger allows free estimation of the intercept, although further assumptions, such as the independence between instrument strength and direct effects, cannot be easily verified. Average directional pleiotropy across genetic variants was assessed from the p-value of the intercept term from MR-Egger (27). Causal estimates in MR Egger are less precise than those obtained by using IVW MR (28). Analysis using MR-Egger has a lower false positive rate but a higher false negative rate than IVW (29).

Further, to assess heterogeneity between individual genetic variant estimates, we used the Q' heterogeneity statistic (30) and the MR pleiotropy residual sum and outlier (MR-PRESSO) test (30). The Q' statistic uses modified 2nd order weights that are a derivation of a Taylor series expansion and take into account uncertainty in both numerator and denominator of the instrumental variable ratio (this eases the no-measurement-error [NOME] assumption) (30). The MR-PRESSO framework relies on the regression of variant-outcome associations on variantexposure associations and implements a global heterogeneity test by comparing the observed distance (residual sums of squares) of all variants to the regression line with the distance expected under the null hypothesis of no pleiotropy (31). In case of evidence of horizontal pleiotropy, the test compares individual variants expected and observed distributions to identify outlier variants. Further we applied on MR-Robust Adjusted Profile Score (RAPS) this method is able to correct for pleiotropy using robust adjusted profile scores. We consider as results, causal estimates that agreed in direction and magnitude across MR methods, pass nominal significance in 
Table 2. Results of the Mendelian Randomization (MR) analysis for effects of serum vitamin D on total, trunk, arm and leg fatfree mass

\begin{tabular}{|c|c|c|c|c|c|c|c|c|c|c|c|}
\hline \multirow[t]{2}{*}{ Exposure } & \multirow[t]{2}{*}{ Outcome } & \multicolumn{4}{|c|}{ MR } & \multicolumn{3}{|c|}{ Heterogeneity } & \multicolumn{3}{|c|}{ Pleiotropy } \\
\hline & & Method & beta & SE & $\mathbf{p}$ & Method & $\mathbf{Q}$ & P-value & Intercept & SE & p \\
\hline \multirow[t]{18}{*}{ Vitamin D (Serum 25(OH)D) } & \multirow{3}{*}{ Total fat-free mass } & MR Egger & 0.019 & 0.039 & 0.657 & MR-Egger & 11.018 & 0.026 & 0.001 & 0.002 & 0.503 \\
\hline & & IVW & 0.042 & 0.02 & 0.038 & IVW & 12.506 & 0.029 & & & \\
\hline & & RAPS & 0.036 & 0.016 & 0.03 & & & & & & \\
\hline & \multirow[t]{3}{*}{ Trunk fat-free mass } & MR Egger & 0.037 & 0.039 & 0.406 & MR-Egger & 11.479 & 0.022 & 0.0004 & 0.002 & 0.817 \\
\hline & & IVW & 0.045 & 0.02 & 0.023 & IVW & 11.655 & 0.04 & & & \\
\hline & & RAPS & 0.039 & 0.017 & 0.019 & & & & & & \\
\hline & \multirow[t]{3}{*}{ Arm fat-free mass (right) } & MR Egger & 0.042 & 0.029 & 0.225 & MR-Egger & 6.415 & 0.17 & 0.0001 & 0.001 & 0.94 \\
\hline & & WM & 0.042 & 0.014 & 0.003 & & & & & & \\
\hline & & IVW & 0.044 & 0.015 & 0.002 & IVW & 6.425 & 0.267 & & & \\
\hline & \multirow{2}{*}{ Arm fat-free mass (left) } & IVW & 0.05 & 0.018 & 0.005 & IVW & 9.499 & 0.091 & & & \\
\hline & & RAPS & 0.046 & 0.016 & 0.005 & & & & & & \\
\hline & \multirow[t]{4}{*}{ Leg fat-free mass (right) } & MR Egger & -0.025 & 0.0401 & 0.561 & MR-Egger & 10.775 & 0.029 & 0.003 & 0.002 & 0.171 \\
\hline & & WM & 0.015 & 0.0152 & 0.334 & & & & & & \\
\hline & & IVW & 0.03 & 0.0258 & 0.238 & IVW & 18.269 & 0.003 & & & \\
\hline & & RAPS & 0.022 & 0.021 & 0.281 & & & & & & \\
\hline & \multirow[t]{3}{*}{ Leg fat-free mass (left) } & MR Egger & -0.008 & 0.0382 & 0.838 & MR-Egger & 9.807 & 0.044 & 0.003 & 0.002 & 0.215 \\
\hline & & WM & 0.022 & 0.0148 & 0.143 & & & & & & \\
\hline & & IVW & 0.039 & 0.0234 & 0.1 & IVW & 15.108 & 0.009 & & & \\
\hline
\end{tabular}

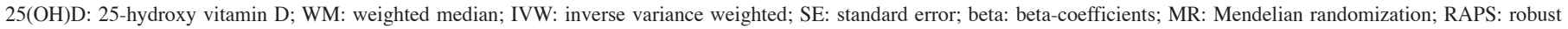
adjusted profile score

IVW MR, and did not show evidence of bias from horizontal pleiotropy using heterogeneity tests. We used $\mathrm{R}$ version 3.4.2 ( $\mathrm{R}$ Core Development Team 2017).

The MR studies assume that the SNPs (instrumental variables) are associated with the outcome only via the exposure (32), so we performed sensitivity analysis excluding SNPs with potentially pleiotropic effects. To assess the instrumental variable analysis "exclusion-restriction" assumption we used Ensembl release (http://useast.ensembl.org/ index.html). Ensembl contains a base of SNP phenotypes.

\section{Ethics}

This investigation uses published or publicly available summary data with no involvement of participants in the study. No original data were collected for this manuscript. Ethical approval for each of the studies included in the investigation can be found in the original publications (including informed consent from each subject).

\section{Results}

In total, 6 SNPs were identified as instrumental variables for serum $25(\mathrm{OH}) \mathrm{D}$, none of which were significantly associated with FFM. A list of all SNP associations is shown in Table 1. The results of MR analysis, displayed as beta-coefficient for interested outcomes per increase in serum $25(\mathrm{OH})$ $\mathrm{D}$, demonstrate a positive and statistically significant effect on total FFM (MR Egger $=\beta: 0.019, p=0.657$ and $I V W=\beta$ : $0.042, \mathrm{p}=0.038$; respectively, Table 2 and Fig. 1), trunk $($ MR Egger $=\beta: 0.037, p=0.406$ and $\mathrm{IVW}=\beta: 0.045, \mathrm{p}=0.023$, respectively, Table 2 and Fig. 1) FFM. This data suggests that each $25 \mathrm{nmol} / \mathrm{L}$ increase in serum $25(\mathrm{OH}) \mathrm{D}$ is associated with an increase of $0.042 \mathrm{~kg}$ of total FFM. Serum $25(\mathrm{OH})$ $\mathrm{D}$ also demonstrated a positive and statistically significant effect on arm FFM (Right arm: MR Egger= $\beta: 0.043, p=0.225$ and IVW= $\beta$ : $0.044, p=0.002$; Left arm: MR Egger $=\beta: 0.033$, $\mathrm{p}=0.398$ and $\mathrm{IVW}=\beta: 0.05, \mathrm{p}=0.005$, respectively, Table 2 . However, results for leg FFM did not demonstrate a statistically significant effect (Right leg: MR Egger $=\beta$ : -0.025 , SE: 0.04, $\mathrm{p}=0.561$ and IVW= $\beta: 0.03$, SE: $0.026, \mathrm{p}=0.238$; Left leg: MR Egger $=\beta:-0.008$, SE: $0.038, p=0.838$ and IVW= $\beta: 0.039$, SE: $0.023, \mathrm{p}=0.1$, respectively, Table 2). 
Figure 1. Scatter plots of the association of the effect of SNP-determined serum 25(OH)D on trunk (A) and total (B) fat-free mass

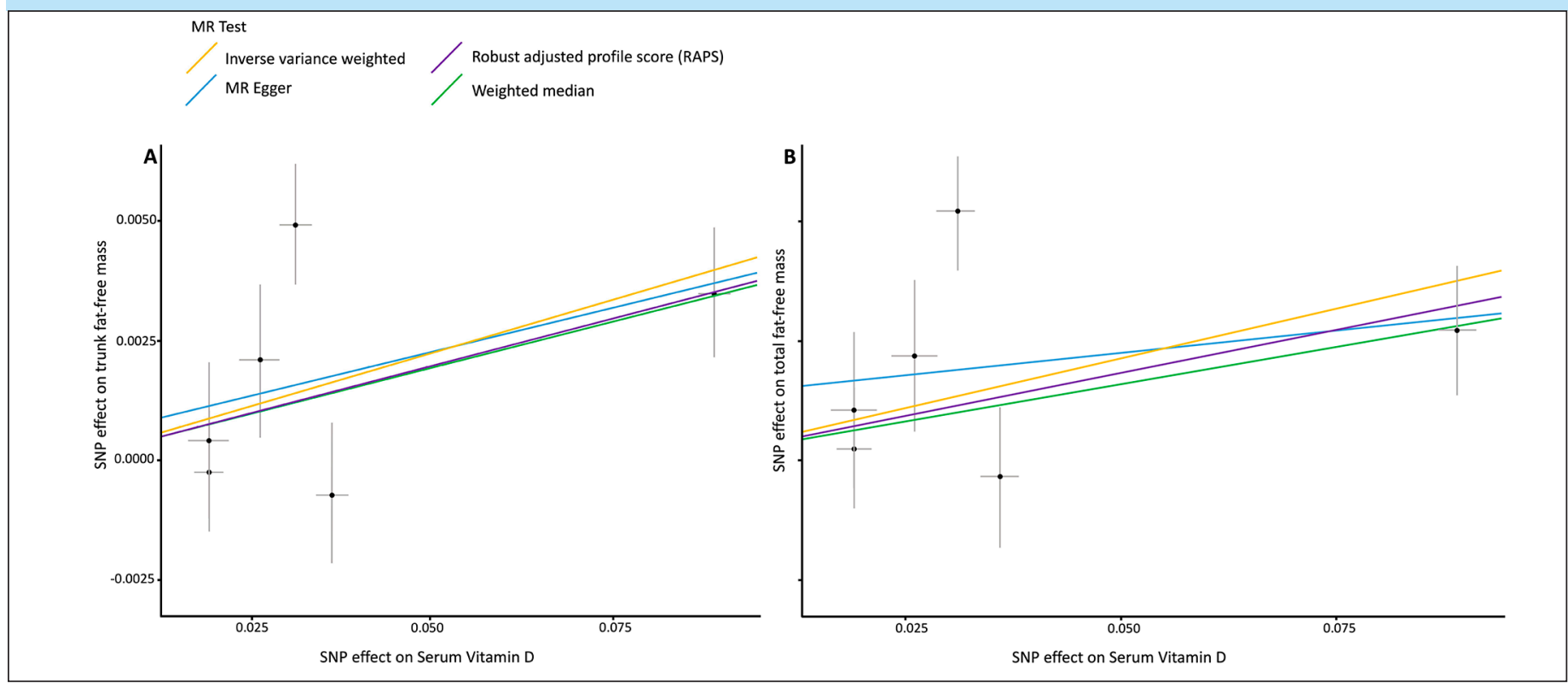

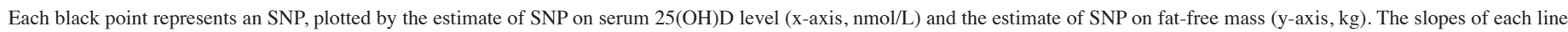
represent the potential causal associations for each method.

The horizontal pleiotropy test, with very negligible Egger regression intercept, also showed a low likelihood of pleiotropy for all our estimations (all p >0.171, Table 2). Further the result of the MR-RAPS was identical with the IVW prediction, which again indicated a statistically low chance of pleiotropy. Heterogeneity tests highlighted no trace of heterogeneity (Table 2). Furthermore, MR-PRESSO analysis did not indicate any outliers for all estimates. Results of leave-one-out method demonstrated that the links are not driven by any single SNP.

\section{Discussion}

Our results illustrate the potentially causal, positive effect of lifetime serum $25(\mathrm{OH}) \mathrm{D}$ concentration on total, trunk and arm FFM. These findings are in agreement with a number of cross-sectional, population-based studies, which have shown a positive relationship between serum $25(\mathrm{OH}) \mathrm{D}$ status and FFM in a wide range of age groups and clinical populations $[3,17,18]$. In a study of 100 adolescents $(15.1 \pm 1.9 \mathrm{y})$, serum $25(\mathrm{OH}) \mathrm{D}$ was positively associated with lean body mass and inversely with fat mass (18). In a cross-sectional study of 127 pre-frail and frail elderly people $(79.0 \pm 7.8 \mathrm{y})$ in The Netherlands, Tieland et al. (3) reported that low 25(OH)D status was associated with reduced muscle mass and poorer physical performance (3). Additionally, a meta-analysis of 12 studies with data from 22,590 individuals (mean range $50-$ $88 \mathrm{yrs}$ ) reported that sarcopenic individuals had lower blood 25(OH)D concentrations than non-sarcopenic controls (17). Conversely, some studies have reported no such association between $25(\mathrm{OH}) \mathrm{D}$ and lean body mass (LBM) or FFM $(19,20)$.

Mechanistically, vitamin D is known to exert its effects of muscle tissue both by regulating expression of target genes via the vitamin D receptor (VDR) and by non-genomic regulation of skeletal muscle intracellular signaling pathways
(33). In animal models, vitamin D supplementation has been demonstrated to activate the mammalian target of rapamycin/ S6 kinase (mTOR/S6K) pathway, which leads to increased muscle protein synthesis (MPS) (34) essential for increases in muscle protein accrual and size (35). Cell culture models have also reported that vitamin D enhances the stimulating effect of leucine and insulin on muscle protein synthesis rates (36) and promotes myogenic differentiation and reduces the expression of myostatin, a known negative regulator of muscle size (37). Vitamin D has also been reported to stimulate the expression of genes involved in the control of cellular growth $(33,38)$. These varied mechanisms may partly explain the adverse effects of low vitamin D status on muscle mass and function.

The present study did not find a statistically significant relationship between genetically determined serum $25(\mathrm{OH}) \mathrm{D}$ concentration and leg FFM. This is not the first study to identify a discrepancy in the relationship between $25(\mathrm{OH}) \mathrm{D}$ status with upper and lower body appendicular lean mass. In a study of frail elderly Dutch people $(n=127$; mean 79 y) $25(\mathrm{OH}) \mathrm{D}$ status was associated with appendicular lean mass (ALM) $(\beta=0.012$ $[\mathrm{P}=0.05])$ but was not significantly associated with leg lean mass $(\beta=0.008[\mathrm{P}=0.08])$ (3). Furthermore, a cross-sectional study of the association of $25(\mathrm{OH}) \mathrm{D}$ status with muscle strength $(\mathrm{n}=419$; healthy men and women; 20-76 y) has also reported a stronger association between $25(\mathrm{OH}) \mathrm{D}$ and muscle strength in the arms compared to the legs (39). One potential explanation for this discrepancy is the reported greater distribution of VDR in type 2 muscle fibres (40) which make up a greater proportion of upper body skeletal muscle (41-44). Vitamin D affects both the diameter and the number of type 2 muscle fibres, which are important for not only young athletes but also the elderly, due to their capacity to reduce the risk of falls, for example (45, 46). Greater expression of VDR has been reported to stimulate muscle hypertrophy through a number of potential mechanisms 
including increased protein synthesis (47). Furthermore, the greater daily utilization of lower extremities, for example, due to locomotion and bearing the individuals body weight during movement, may provide a superior stimulus for muscle hypertrophy. Further research is clearly needed to elucidate the mechanisms by which vitamin D differentially affects lower and upper body appendicular muscle physiology.

This study highlights the importance of serum vitamin D concentrations in accruing and maintaining FFM, which itself is associated with lower risk of frailty and mortality $(7,11,14)$. Addressing vitamin D insufficiency is challenging as the main source of vitamin D in humans is sun exposure (48) which is unlikely to become a widely accepted and implemented strategy. Furthermore, dietary intakes of vitamin D are typically low (49) due to low levels in common foodstuffs (50). Therefore, at a population level, food fortification with vitamin $\mathrm{D}$, and at an individual level, supplementation may be the most effective methods to increase 25(OH)D status to sufficient levels (5).

A major strength of our study was the large sample population study with access to individual participant data of high validity and with the relevant SNPs available for both $25(\mathrm{OH}) \mathrm{D}$ serum concentration and FFM. Furthermore, the use of the Mendelian randomisation approach allowed us to examine the potential causal effects of serum $25(\mathrm{OH}) \mathrm{D}$, largely without the disadvantages of confounding or reverse causation.

A potential limitation of this study is the use of segmental bioelectrical impedance analysis (BIA) as the method for determining FFM in the UK Biobank cohort. The accuracy of BIA measurement is known to be affected by hydration status; however the UK Biobank protocol did not specify any procedures to standardise some determinants of hydration before the assessment. This could potentially lead to inaccuracies in the values attained for FFM (51). Furthermore, evidence suggests that BIA is less accurate at high BMI levels (52) and considering the range of BMI included in the UK Biobank cohort, this should be taken into consideration with these results.

\section{Conclusions}

Evidence for a potentially causal association of serum 25(OH)D with total, trunk and arm FFM was found. However, the relationship between serum 25(OH)D and leg FFM was not statistically significant. This finding highlights the importance of maintaining sufficient $25(\mathrm{OH}) \mathrm{D}$ status throughout the life course in order to maintain adequate lean mass, a factor associated with multiple chronic disease. Future research should address the causal role and potential mechanisms of serum $25(\mathrm{OH}) \mathrm{D}$ on FFM accrual and maintenance as well as the apparent lack of effect on leg FFM.

Availability of data and material: Not applicable.

Code availability: Not applicable.

Consent for publication: All the authors have read and approved the revised manuscript, and they are willing to publish it.
Author contributions: RK drafted the manuscript. MM conceived the study, acquired the data and performed the analyses. RK, MM, MI and IGD interpreted the findings. All authors contributed to critical reading and revision of the draft report. All authors approved the final version to be published.

Conflict of interest: RK has received a speaker honorarium for a symposium hosted by the British Association for Parenteral and Enteral Nutrition. RK has received payment from Myprotein UK for the production of educational media content. MM, MI and IGD declare no conflict of interest.

Funding: RK is a beneficiary of a postgraduate stipend from the Institute for Health Research from Liverpool John Moores University. MI, IGD and MM declare that the received no financial assistance in support of the study.

Open Access: This article is distributed under the terms of the Creative Commons Attribution 4.0 International License (http://creativecommons.org/licenses/by/4.0/), which permits use, duplication, adaptation, distribution and reproduction in any medium or format, as long as you give appropriate credit to the original author(s) and the source, provide a link to the Creative Commons license and indicate if changes were made.

\section{References}

1. Wang H, Chen W, Li D, Yin X, Zhang X, Olsen N, Zheng SG. Vitamin D and Chronic Diseases. Aging Dis 2017;8 (3):346-353. doi:10.14336/ad.2016.1021

2. Girgis CM. Vitamin D and Skeletal Muscle: Emerging Roles in Development, Anabolism and Repair. Calcif Tissue Int 2020;106 (1):47-57. doi:10.1007/s00223-01900583-4

3. Tieland M, Brouwer-Brolsma EM, Nienaber-Rousseau C, van Loon LJC, De Groot LCPGM. Low vitamin D status is associated with reduced muscle mass and impaired physical performance in frail elderly people. European Journal of Clinical Nutrition 2013;67 (10):1050-1055. doi:10.1038/ejen.2013.144

4. Cashman KD, Dowling KG, Škrabáková Z, Gonzalez-Gross M, Valtueña J, De Henauw S, Moreno L, Damsgaard CT, Michaelsen KF, Mølgaard C, Jorde R, Grimnes G, Moschonis G, Mavrogianni C, Manios Y, Thamm M, Mensink GB, Rabenberg M, Busch MA, Cox L, Meadows S, Goldberg G, Prentice A, Dekker JM, Nijpels G, Pilz S, Swart KM, van Schoor NM, Lips P, Eiriksdottir G, Gudnason V, Cotch MF, Koskinen S, Lamberg-Allardt C, Durazo-Arvizu RA, Sempos CT, Kiely M. Vitamin D deficiency in Europe: pandemic? Am J Clin Nutr 2016;103 (4):1033-1044. doi:10.3945/ajen.115.120873

5. Cashman KD. Vitamin D Deficiency: Defining, Prevalence, Causes, and Strategies of Addressing. Calcif Tissue Int 2020;106 (1):14-29. doi:10.1007/s00223-019-00559-4

6. Del Valle HB, Yaktine AL, Taylor CL, Ross AC. Dietary reference intakes for calcium and vitamin D. 2011

7. Xu W, Chen T, Cai Y, Hu Y, Fan L, Wu C. Sarcopenia in Community-Dwelling Oldest Old is Associated with Disability and Poor Physical Function. The journal of nutrition, health \& aging 2020;24 (3):339-345. doi:10.1007/s12603-020-1325-4

8. Cruz-Jentoft AJ, Bahat G, Bauer J, Boirie Y, Bruyere O, Cederholm T, Cooper C, Landi F, Rolland Y, Sayer AA, Schneider SM, Sieber CC, Topinkova E, Vandewoude M, Visser M, Zamboni M. Sarcopenia: revised European consensus on definition and diagnosis. Age Ageing 2019;48 (1):16-31. doi:10.1093/ageing/afy169

9. Bahat G, İlhan B. Sarcopenia and the cardiometabolic syndrome: A narrative review. European Geriatric Medicine 2016;7 (3):220-223. doi:https://doi.org/10.1016/j. eurger.2015.12.012

10. Scott D, de Courten B, Ebeling PR. Sarcopenia: a potential cause and consequence of type 2 diabetes in Australia's ageing population? The Medical journal of Australia 2016;205 (7):329-333

11. Schaap LA, van Schoor NM, Lips P, Visser M. Associations of Sarcopenia Definitions, and Their Components, With the Incidence of Recurrent Falling and Fractures: The Longitudinal Aging Study Amsterdam. J Gerontol A Biol Sci Med Sci 2018;73 (9):1199-1204. doi:10.1093/gerona/glx245

12. Hayashi T, Umegaki H, Makino T, Cheng XW, Shimada H, Kuzuya M. Association between sarcopenia and depressive mood in urban-dwelling older adults: A crosssectional study. Geriatr Gerontol Int 2019;19 (6):508-512. doi:10.1111/ggi.13650

13. Hsu YH, Liang CK, Chou MY, Liao MC, Lin YT, Chen LK, Lo YK. Association of cognitive impairment, depressive symptoms and sarcopenia among healthy older men in the veterans retirement community in southern Taiwan: a cross-sectional study. Geriatr Gerontol Int 2014;14 Suppl 1:102-108. doi:10.1111/ggi.12221

14. Abramowitz MK, Hall CB, Amodu A, Sharma D, Androga L, Hawkins M. Muscle mass, BMI, and mortality among adults in the United States: A population-based cohort study. PLoS One 2018;13 (4):e0194697. doi:10.1371/journal.pone.0194697

15. Webb AR, Pilbeam C, Hanafin N, Holick MF. An evaluation of the relative contributions of exposure to sunlight and of diet to the circulating concentrations of 25-hydroxyvitamin D in an elderly nursing home population in Boston. Am J Clin Nutr 1990;51 (6):1075-1081. doi:10.1093/ajen/51.6.1075

16. Whitmore SE. Vitamin D deficiency in homebound elderly persons. Jama 1996;275 (11):838-839

17. Luo J, Quan Z, Lin S, Cui L. The association between blood concentration of 25 hydroxyvitamin D and sarcopenia: a meta-analysis. Asia Pac J Clin Nutr 2018;27 
(6):1258-1270. doi:10.6133/apjen.201811_27(6).0013

18. Wierzbicka E, Szalecki M, Pludowski P, Jaworski M, Brzozowska A. Vitamin D status, body composition and glycemic control in Polish adolescents with type 1 diabetes. Minerva Endocrinol 2016;41 (4):445-455

19. Ceglia L, Chiu GR, Harris SS, Araujo AB. Serum 25-hydroxyvitamin D concentration and physical function in adult men. Clinical Endocrinology 2011;74 (3):370-376. doi:https://doi.org/10.1111/j.1365-2265.2010.03926.x

20. De Pergola G, Martino T, Zupo R, Caccavo D, Pecorella C, Paradiso S, Silvestris F, Triggiani V. 25 Hydroxyvitamin D Levels are Negatively and Independently Associated with Fat Mass in a Cohort of Healthy Overweight and Obese Subjects. Endocr Metab Immune Disord Drug Targets 2019;19 (6):838-844. doi:10.2174/187153 0319666190122094039

21. Larsson SC. Mendelian randomization as a tool for causal inference in human nutrition and metabolism. Current opinion in lipidology 2021;32 (1):1-8. doi:10.1097/ mol.0000000000000721

22. Smith GD, Ebrahim S. 'Mendelian randomization': can genetic epidemiology contribute to understanding environmental determinants of disease? Int $\mathbf{J}$ Epidemiol 2003;32 (1):1-22. doi:10.1093/ije/dyg070

23. Plotnikov D, Guggenheim JA. Mendelian randomisation and the goal of inferring causation from observational studies in the vision sciences. Ophthalmic and Physiological Optics 2019;39 (1):11-25. doi:10.1111/opo.12596

24. Jiang X, O'Reilly PF, Aschard H, Hsu YH, Richards JB, Dupuis J, Ingelsson E, Karasik D, Pilz S, Berry D, Kestenbaum B, Zheng J, Luan J, Sofianopoulou E, Streeten EA, Albanes D, Lutsey PL, Yao L, Tang W, Econs MJ, Wallaschofski H, Volzke H, Zhou A, Power C, McCarthy MI, Michos ED, Boerwinkle E, Weinstein SJ, Freedman ND, Huang WY, Van Schoor NM, van der Velde N, Groot L, Enneman A, Cupples LA, Booth SL, Vasan RS, Liu CT, Zhou Y, Ripatti S, Ohlsson C, Vandenput L, Lorentzon M, Eriksson JG, Shea MK, Houston DK, Kritchevsky SB Liu Y, Lohman KK, Ferrucci L, Peacock M, Gieger C, Beekman M, Slagboom E, Deelen J, Heemst DV, Kleber ME, Marz W, de Boer IH, Wood AC, Rotter JI, Rich SS, Robinson-Cohen C, den Heijer M, Jarvelin MR, Cavadino A, Joshi PK, Wilson JF, Hayward C, Lind L, Michaelsson K, Trompet S, Zillikens MC, Uitterlinden AG, Rivadeneira F, Broer L, Zgaga L, Campbell H, Theodoratou E, Farrington SM, Timofeeva M, Dunlop MG, Valdes AM, Tikkanen E, Lehtimaki T, Lyytikainen LP, Kahonen M, Raitakari OT, Mikkila V, Ikram MA, Sattar N, Jukema JW, Wareham NJ, Langenberg C, Forouhi NG, Gundersen TE, Khaw KT, Butterworth AS, Danesh J, Spector T, Wang TJ, Hypponen E, Kraft P, Kiel DP. Genome-wide association study in 79,366 European-ancestry individuals informs the genetic architecture of 25-hydroxyvitamin D levels. Nature communications 2018;9 (1):260. doi:10.1038/ s41467-017-02662-2

25. UK Biobank-Body Composition Measurement. 2011. https://biobank.ctsu.ox.ac.uk/ showcase/showcase/docs/body_composition.pdf. Accessed August 15, 2021

26. Sudlow C, Gallacher J, Allen N, Beral V, Burton P, Danesh J, Downey P, Elliott P, Green J, Landray M. UK biobank: an open access resource for identifying the causes of a wide range of complex diseases of middle and old age. PLoS medicine 2015;12 (3):e1001779

27. Bowden J, Davey Smith G, Haycock PC, Burgess S. Consistent Estimation in Mendelian Randomization with Some Invalid Instruments Using a Weighted Median Estimator. Genetic epidemiology 2016;40 (4):304-314. doi:10.1002/gepi.21965

28. Bowden J, Davey Smith G, Burgess S. Mendelian randomization with invalid instruments: effect estimation and bias detection through Egger regression. International journal of epidemiology 2015;44 (2):512-525. doi:10.1093/ije/dyv080

29. Burgess S, Bowden J, Fall T, Ingelsson E, Thompson SG. Sensitivity Analyses for Robust Causal Inference from Mendelian Randomization Analyses with Multiple Genetic Variants. Epidemiology (Cambridge, Mass) 2017;28 (1):30-42. doi:10.1097/ ede. 0000000000000559

30. Bowden J, Del Greco MF, Minelli C, Davey Smith G, Sheehan N, Thompson J. A framework for the investigation of pleiotropy in two-sample summary data Mendelian randomization. Statistics in medicine 2017;36 (11):1783-1802. doi:10.1002/sim.7221

31. Verbanck M, Chen CY, Neale B, Do R. Detection of widespread horizontal pleiotropy in causal relationships inferred from Mendelian randomization between complex traits and diseases. Nature genetics 2018;50 (5):693-698. doi:10.1038/s41588-018-0099-7

32. Lawlor DA, Harbord RM, Sterne JA, Timpson N, Davey Smith G. Mendelian randomization: using genes as instruments for making causal inferences in epidemiology. Statistics in medicine 2008;27 (8):1133-1163. doi:10.1002/sim.3034
33. Boland RL. VDR activation of intracellular signaling pathways in skeletal muscle. Mol Cell Endocrinol 2011;347 (1-2):11-16. doi:10.1016/j.mce.2011.05.021

34. Vignale K, Greene ES, Caldas JV, England JA, Boonsinchai N, Sodsee P, Pollock ED, Dridi S, Coon CN. 25-Hydroxycholecalciferol Enhances Male Broiler Breast Meat Yield through the mTOR Pathway. The Journal of nutrition 2015;145 (5):855-863. doi:10.3945/jn.114.207936

35. Atherton PJ, Smith K. Muscle protein synthesis in response to nutrition and exercise. The Journal of physiology 2012;590 (5):1049-1057. doi:10.1113/jphysiol.2011.225003

36. Salles J, Chanet A, Giraudet C, Patrac V, Pierre P, Jourdan M, Luiking YC, Verlaan S Migné C, Boirie Y, Walrand S. 1,25(OH)2-vitamin D3 enhances the stimulating effect of leucine and insulin on protein synthesis rate through Akt/PKB and mTOR mediated pathways in murine C2C12 skeletal myotubes. Mol Nutr Food Res 2013;57 (12):21372146. doi:10.1002/mnfr.201300074

37. Garcia LA, King KK, Ferrini MG, Norris KC, Artaza JN. 1,25(OH)2vitamin D3 stimulates myogenic differentiation by inhibiting cell proliferation and modulating the expression of promyogenic growth factors and myostatin in $\mathrm{C} 2 \mathrm{C} 12$ skeletal muscle cells. Endocrinology 2011;152 (8):2976-2986. doi:10.1210/en.2011-0159

38. Neary J. MAPK Cascades in Cell Growth and Death. Physiology 1997;12 (6):286-293 doi:10.1152/physiologyonline.1997.12.6.286

39. Grimaldi AS, Parker BA, Capizzi JA, Clarkson PM, Pescatello LS, White MC, Thompson PD. 25(OH) vitamin D is associated with greater muscle strength in healthy men and women. Medicine and science in sports and exercise 2013;45 (1):157-162. doi:10.1249/MSS.0b013e31826c9a78

40. Srikuea R, Hirunsai M, Charoenphandhu N. Regulation of vitamin D system in skeletal muscle and resident myogenic stem cell during development, maturation, and ageing. Sci Rep 2020;10 (1):8239. doi:10.1038/s41598-020-65067-0

41. Ørtenblad N, Nielsen J, Boushel R, Söderlund K, Saltin B, Holmberg HC. The Muscle Fiber Profiles, Mitochondrial Content, and Enzyme Activities of the Exceptionally Well-Trained Arm and Leg Muscles of Elite Cross-Country Skiers. Front Physiol 2018;9:1031. doi:10.3389/fphys.2018.01031

42. Johnson MA, Polgar J, Weightman D, Appleton D. Data on the distribution of fibre types in thirty-six human muscles. An autopsy study. J Neurol Sci 1973;18 (1):111129. doi:10.1016/0022-510x(73)90023-3

43. Klein CS, Marsh GD, Petrella RJ, Rice CL. Muscle fiber number in the biceps brachii muscle of young and old men. Muscle \& nerve 2003;28 (1):62-68. doi:10.1002/ mus.10386

44. Travnik L, Pernus F, Erzen I. Histochemical and morphometric characteristics of the normal human vastus medialis longus and vastus medialis obliquus muscles. J Anat 1995;187 ( Pt 2) (Pt 2):403-411

45. Remelli F, Vitali A, Zurlo A, Volpato S. Vitamin D Deficiency and Sarcopenia in Older Persons. Nutrients 2019;11 (12). doi:10.3390/nu11122861

46. Koundourakis NE, Avgoustinaki PD, Malliaraki N, Margioris AN. Muscular effects of vitamin $\mathrm{D}$ in young athletes and non-athletes and in the elderly. Hormones (Athens) 2016;15 (4):471-488. doi:10.14310/horm.2002.1705

47. Bass JJ, Nakhuda A, Deane CS, Brook MS, Wilkinson DJ, Phillips BE, Philp A, Tarum J, Kadi F, Andersen D, Garcia AM, Smith K, Gallagher IJ, Szewczyk NJ, Cleasby ME, Atherton PJ. Overexpression of the vitamin D receptor (VDR) induces skeletal muscle hypertrophy. Mol Metab 2020;42:101059. doi:10.1016/j.molmet.2020.101059

48. Engelsen O. The Relationship between Ultraviolet Radiation Exposure and Vitamin D Status. Nutrients 2010;2 (5):482-495

49. Kiely M, Black LJ. Dietary strategies to maintain adequacy of circulating 25-hydroxyvitamin D concentrations. Scand J Clin Lab Invest Suppl 2012;243:14-23. doi:10.3109/00365513.2012.681893

50. Schmid A, Walther B. Natural Vitamin D Content in Animal Products. Advances in Nutrition 2013;4 (4):453-462. doi:10.3945/an.113.003780

51. Kyle UG, Bosaeus I, De Lorenzo AD, Deurenberg P, Elia M, Manuel Gómez J, Lilienthal Heitmann B, Kent-Smith L, Melchior JC, Pirlich M, Scharfetter H, A MWJS, Pichard C. Bioelectrical impedance analysis-part II: utilization in clinical practice. Clinical nutrition (Edinburgh, Scotland) 2004;23 (6):1430-1453 doi:10.1016/j.clnu.2004.09.012

52. Neovius M, Hemmingsson E, Freyschuss B, Uddén J. Bioelectrical impedance underestimates total and truncal fatness in abdominally obese women. Obesity (Silver Spring) 2006;14 (10):1731-1738. doi:10.1038/oby.2006.199

How to cite this article: R. Kirwan, M. Isanejad, I.G. Davies, et al. Genetically Determined Serum 25-Hydroxyvitamin D Is Associated with Total, Trunk, and Arm Fat-Free Mass: A Mendelian Randomization Study. J Nutr Health Aging. 2022;26(1):4651, http://dx.doi.org/10.1007/s12603-021-1696-1 